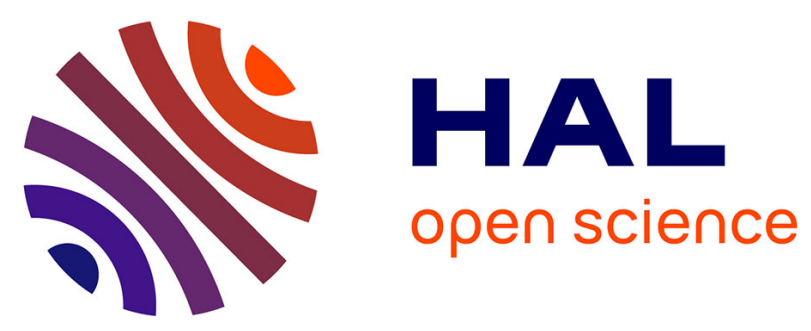

\title{
Magnetic Field-Induced Phase Transition in the Quasi Two-Dimensional Organic Superconductor (BEDO-TTF)2ReO4 .H2O
}

\author{
A. Audouard, V. Laukhin, Cyril Proust, L. Brossard, N. Kushch
}

\section{- To cite this version:}

A. Audouard, V. Laukhin, Cyril Proust, L. Brossard, N. Kushch. Magnetic Field-Induced Phase Transition in the Quasi Two-Dimensional Organic Superconductor (BEDO-TTF)2ReO4 .H2O. Journal de Physique I, 1997, 7 (4), pp.599-610. 10.1051/jp1:1997178 • jpa-00247347

HAL Id: jpa-00247347

https://hal.science/jpa-00247347

Submitted on 1 Jan 1997

HAL is a multi-disciplinary open access archive for the deposit and dissemination of scientific research documents, whether they are published or not. The documents may come from teaching and research institutions in France or abroad, or from public or private research centers.
L'archive ouverte pluridisciplinaire HAL, est destinée au dépôt et à la diffusion de documents scientifiques de niveau recherche, publiés ou non, émanant des établissements d'enseignement et de recherche français ou étrangers, des laboratoires publics ou privés. 


\title{
Magnetic Field-Induced Phase Transition in the Quasi Two-Dimensional Organic Superconductor $(\mathrm{BEDO}-\mathrm{TTF})_{2} \mathrm{ReO}_{4} \cdot \mathrm{H}_{2} \mathrm{O}$
}

\author{
A. Audouard $\left({ }^{1, *}\right)$, V.N. Laukhin $\left({ }^{2, * *}\right)$, C. Proust $\left({ }^{1}\right)$, L. Brossard $\left({ }^{1}\right)$ \\ and N.D. Kushch $\left({ }^{2}\right)$ \\ $\left({ }^{1}\right)$ Laboratoire de Physique de la Matière Condensée $\left({ }^{* *}\right)$, \\ Service National des Champs Magnétiques Pulsés $\left({ }^{* * * *}\right)$, INSA, \\ Complexe Scientifique de Rangueil, 31077 Toulouse, France \\ $\left({ }^{2}\right)$ Institute of Chemical Physics in Chernogolovka, Russian Academy of Sciences, \\ Chernogolovka, MD 142432, Russia
}

(Received 3 October 1996, accepted 19 December 1996)

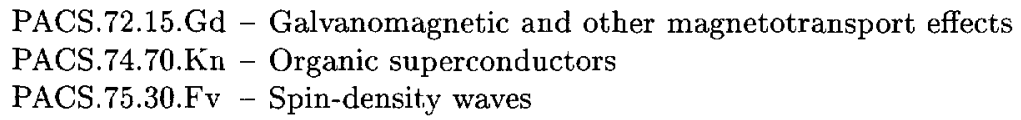

\begin{abstract}
Transverse magnetoresistance of the two-dimensional (2D) organic superconductor (BEDO-TTF) $)_{2} \mathrm{ReO}_{4} \cdot \mathrm{H}_{2} \mathrm{O}$ has been measured in pulsed magnetic fields up to $52 \mathrm{~T}$ and in the temperature range from $1.7 \mathrm{~K}$ to $4.2 \mathrm{~K}$. For magnetic field lower than $\sim 15 \mathrm{~T}$, applied perpendicularly to the conducting planes, the data are in good agreement with previous studies, namely, the two Shubnikov-de Haas oscillation series with frequency $F_{1}=(38 \pm 2) \mathrm{T}$ and $F_{2}=(74 \pm 2) \mathrm{T}$ attributed to electron and hole orbits, respectively, are observed. However, as the magnetic field is rotated in the plane perpendicular to the best conductivity direction, the behaviour of the oscillations is not consistent with a perfect $2 \mathrm{D}$ picture. In addition, a new oscillation series with frequency about twice $F_{2}\left(F_{4}=(150 \pm 10) \mathrm{T}\right)$ rises up at high magnetic field while the amplitude of the oscillations with the $F_{2}$ frequency decreases. Furthermore, magnetic fields higher than $10 \mathrm{~T}$. applied parallel to the conducting planes, yield negative magnetoresistance. These features are discussed on the basis of the available Fermi surface calculations.
\end{abstract}

\section{Introduction}

Due to their low dimensionality, the low temperature state of organic compounds is sensitive to applied pressure and magnetic field. In that respect, Bechgaard salts (TMTSF) ${ }_{2} \mathrm{X}$ with $\mathrm{X}=\mathrm{PF}_{6}, \mathrm{ClO}_{4}, \mathrm{NO}_{3}$, etc. have been extensively studied [1]. Roughly speaking, it is generally admitted that pressure and magnetic field have opposite effect in these compounds. Indeed, while an applied pressure destroys the spin density wave (SDW) ground state of e.g. (TMTSF) ${ }_{2} \mathrm{PF}_{6}$ [2], a magnetic field applied parallel to the less conducting direction improves

$\left({ }^{*}\right)$ Author for correspondence (e-mail: audouard@insatlse.insa-tlse.fr)

$\left(^{(*)}\right.$ Present address: Institut de Ciencia de Materials de Barcelona (CSIC), Campus de la UAB, 08193 Bellaterra, Spain

$\left({ }^{* * *}\right)$ UMR CNRS 5646

$\left({ }^{* * * *}\right)$ UMS CNRS 5642 
the nesting of the quasi one-dimensional (1D) sheets of the Fermi surface (FS). This leads to e.g. the field-induced spin density wave phenomenon in (TMTSF) ${ }_{2} \mathrm{ClO}_{4}$ [3] or to the stabilisation of the SDW state in (TMTSF) ${ }_{2} \mathrm{NO}_{3}[4]$ at ambient pressure, in agreement with theoretical predictions $[5,6]$. In contrast, the behaviour of the $\alpha-(\mathrm{BEDT}-\mathrm{TTF})_{2} \mathrm{MHg}(\mathrm{XCN})_{4}$ compounds (where $\mathrm{M}=\mathrm{K} . \mathrm{Tl}, \mathrm{Rb}, \mathrm{NH}_{4} ; \mathrm{X}=\mathrm{S}$, Se), under pressure and magnetic field, is very different. It is worth to note that, although the FS is very similar for all the members of this family and is composed of both a close $2 \mathrm{D}$ orbit and $1 \mathrm{D}$ sheets, they exhibit different ground states. Indeed, the compound with $\mathrm{M} / \mathrm{X}=\mathrm{NH}_{4} / \mathrm{S}$ undergoes a superconducting transition at ambient pressure which is destroyed by increases in pressure [7] while the compound with $\mathrm{M} / \mathrm{X}=\mathrm{K} / \mathrm{S}$ exhibits a SDW ground state which is suppressed by a magnetic field higher than $\sim 22 \mathrm{~T}[8]$. It can be noticed that the brother compound of the latter, with $\mathrm{M} / \mathrm{X}=\mathrm{K} / \mathrm{Se}$, reveals a metallic ground state without neither SDW nor superconductivity. Finally, another kind of behaviour has recently been observed in $\beta^{\prime \prime}-(\mathrm{BEDT}-\mathrm{TTF})_{2} \mathrm{AuBr}_{2}$ which has been shown to undergo a field-induced transition between two different SDW states at $\sim 15 \mathrm{~T}[9]$.

The FS of the (BEDO-TTF) ${ }_{2} \mathrm{ReO}_{4} \cdot \mathrm{H}_{2} \mathrm{O}$ compound is peculiar when compared to other organic compounds. Indeed, according to band structure calculations [10], the room temperature FS consists in one hole and two electron tubes. their cross section area amounting to 3.4 and $1.7 \%$ of the first Brillouin zone (FBZ) area, respectively. According to these calculations, this compound should be regarded as a $2 \mathrm{D}$ compensated semi-metal. Nevertheless, Hall effect measurements have evidenced the dominant contribution of the hole carriers to the conductivity [10] which exhibits a metallic behaviour down to $\sim 35 \mathrm{~K}$ although several phase transitions occur as the temperature decreases [10-13]. In the lower temperature range, from 35 to $2.4 \mathrm{~K}$, an increase of the resistance is observed due to the condensation of a SDW state [12]. However, this SDW state is not very stable since it is (i) overcome by a superconducting state at $2.4 \mathrm{~K}[11,12]$, and (ii) suppressed by a rather low (1 kbar) hydrostatic pressure [13]. Magnetoresistance experiments performed at ambient pressure on this compound have revealed two Shubnikov-de Haas (SdH) oscillations series linked to cylindrical carrier tubes. Their axis is perpendicular to the conducting planes [10] and their extremal cross section area is equal to $0.7 \%$ [10] and $1.5-1.6 \%[10,14]$ of the FBZ area. These two series have been respectively attributed to electron and hole carriers [10] although their respective frequency, hereafter identified as $F_{1}$ and $F_{2}$, yields cyclotron orbits areas lower, by a factor of more than two, than those predicted by room temperature band structure calculations. Experiments under hydrostatic pressure up to $11 \mathrm{kbar}$ and magnetic fields up to $12 \mathrm{~T}$ have evidenced the onset of a phase transition towards a less conducting state at temperatures below $4 \mathrm{~K} \mathrm{[15].} \mathrm{However,} \mathrm{nothing} \mathrm{is} \mathrm{known} \mathrm{on} \mathrm{the}$ magnetic field sensitivity of the ambient pressure SDW state. The aim of this paper is to explore the effect of a high magnetic field (up to $52 \mathrm{~T}$ ) on (BEDO-TTF) ${ }_{2} \mathrm{ReO}_{4} \cdot \mathrm{H}_{2} \mathrm{O}$, in the experimental conditions where both the compound is in the SDW state and $\mathrm{SdH}$ oscillations can be observed.

\section{Experimental}

The details of the synthesis of $(\mathrm{BEDO}-\mathrm{TTF})_{2} \mathrm{ReO}_{4} \cdot \mathrm{H}_{2} \mathrm{O}$ single crystals are given in [12]. Structural data were reported in $[11,12,16]$. The sample was mounted using the standard four-contact method as reported in [15]. Alternating current $(100 \mu \mathrm{A}, 50 \mathrm{kHz})$ was applied along the best conduction direction $c$ while the magnetic field was applied perpendicularly to c. The signal across the potential contacts was detected by a lock-in amplifier. The single crystal used for measurements had dimensions $(2 \times 0.4 \times 0.05) \mathrm{mm}^{3}$ and its room temperature conductivity was about $30 \Omega^{-1} \mathrm{~cm}^{-1}$ A sample holder rotating around the $c$ axis allowed a $180^{\circ}$ variation of the angle $\theta$ between the magnetic field direction and the normal $\left(b^{*}\right)$ to 


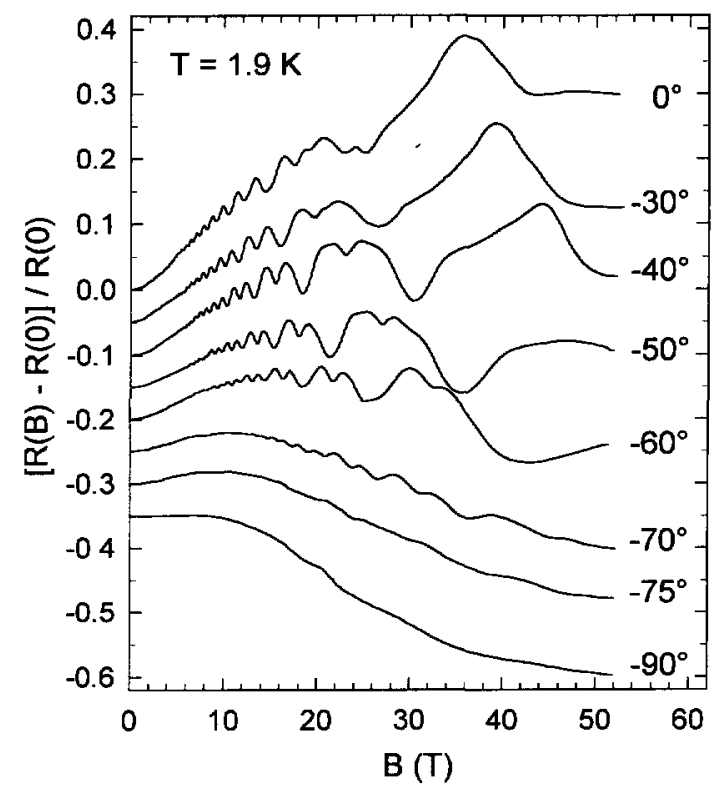

Fig. 1. - Magnetoresistance data of (BEDO-TTF $)_{2} \mathrm{ReO}_{4} \cdot \mathrm{H}_{2} \mathrm{O}$ at $1.9 \mathrm{~K}$ for some magnetic field orientations. $\theta$ is the $\left(B, b^{*}\right)$ angle.

the conducting ac plane. Transverse magnetoresistance data were recorded during the decay of pulsed magnetic field. The maximum field was $52 \mathrm{~T}$; the raising time and the pulse duration were 37 and $250 \mathrm{~ms}$, respectively. Temperature was varied between 1.7 and $4.2 \mathrm{~K}$, as measured by a calibrated rhodium-iron sensor.

\section{Results and Analysis}

Figure 1 displays magnetoresistance data recorded at $1.9 \mathrm{~K}$ for several field directions. It can be remarked first that the monotonous part of the magnetoresistance exhibits a saturating behaviour for angles $\theta<50^{\circ}$ and even decreases at high magnetic field for $\theta \geq 50^{\circ}$. Furthermore, for $\theta=90^{\circ}$, it starts with a nearly zero-slope and exhibits, above $10 \mathrm{~T}$, a negative magnetoresistance which tends to saturate at high magnetic field. It will be shown in the light of the analysis of the oscillatory part of the magnetoresistance that this feature is a signature of a field-induced phase transition towards a more conducting state.

Secondly, data in Figure 1 also exhibit magnetoresistance oscillations, as already reported in previous studies $[10,14,15]$. The oscillatory magnetoresistance has been calculated by dividing the resistance by its monotonous part $\left(R_{\text {background }}\right)$. Data for $\theta=0$ are shown in Figure 2 . Drastic changes in the oscillation spectrum along with the magnetic field is revealed by this figure. Indeed, two oscillations series (hereafter referred as $S_{1}$ and $S_{2}$ ) can be identified at low magnetic field. The frequency of these oscillations, deduced from Fourier transform (FT) calculations (see Fig. 3), is $F_{1}=(38 \pm 2) \mathrm{T}$ and $F_{2}=(74 \pm 2) \mathrm{T}$, respectively for $\mathrm{S}_{1}$ and $\mathrm{S}_{2}$ series. These values are in agreement with data of references $[10]\left(F_{1}=(37 \pm 3) \mathrm{T}, F_{2}=(76 \pm 2) \mathrm{T}\right)$ and [14] $\left(F_{2}=81 \mathrm{~T}\right)$. Recall that $\mathrm{S}_{1}$ and $\mathrm{S}_{2}$ series have respectively been attributed to electron and hole carriers [10]. Data in Figure 2 can be accounted for by the SdH model, assuming 


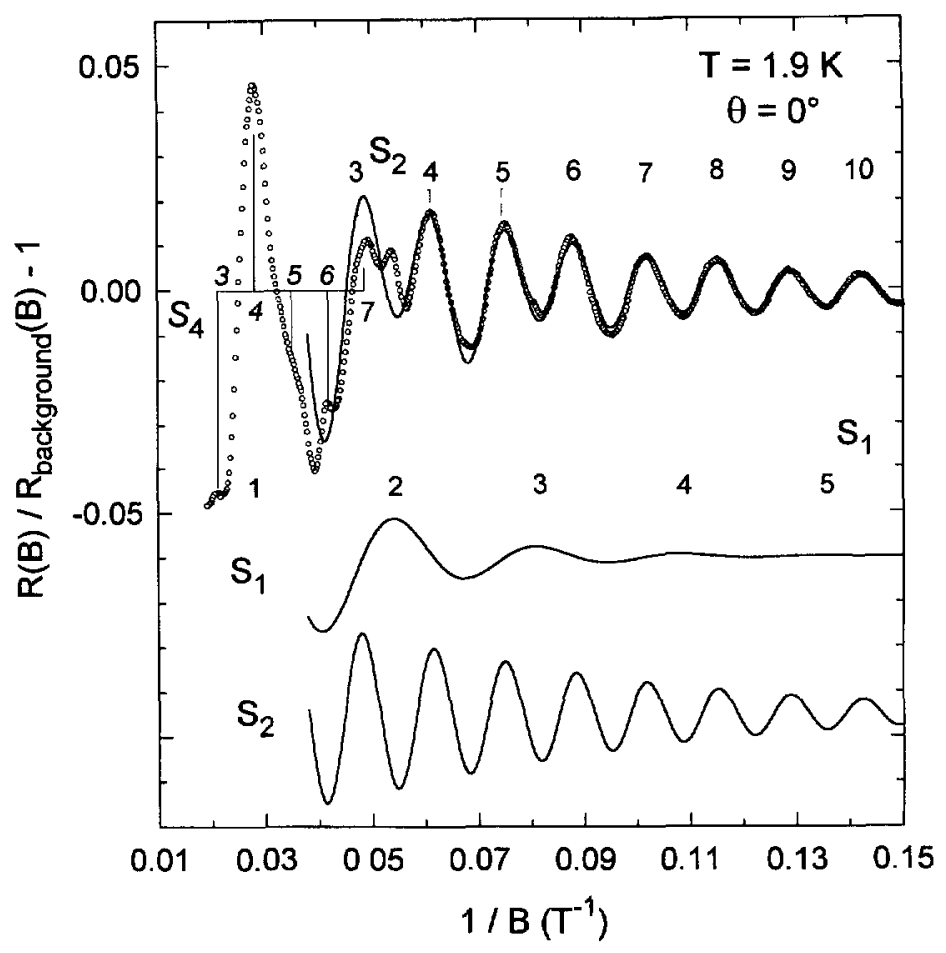

Fig. 2. - Oscillatory part of the magnetoresistance of (BEDO-TTF) ${ }_{2} \mathrm{ReO}_{4} \cdot \mathrm{H}_{2} \mathrm{O}$ at $1.9 \mathrm{~K}$ deduced from Figure 1 for $B \| b^{*}$. $R_{\text {background }}$ is the monotonous part of the field-dependent resistance. The upper solid line is the best fit of the $\mathrm{SdH}$ model (cf. Eq. (1)) to the data in the low magnetic field range (see text). The respective contributions of $S_{1}$ and $S_{2}$ series in the fit are also displayed.

the oscillatory magnetoresistance is the sum of two $\mathrm{SdH}$ oscillations series:

$$
\frac{R(B)}{R_{\text {background }}}=1+\sum_{\imath=1}^{2} a_{\imath} \frac{u_{0} T m_{\mathrm{c} \imath} / B^{1 / 2}}{\sinh \left(u_{0} T m_{\mathrm{c} \imath} / B\right)} \exp \left(-u_{0} T_{\mathrm{D}} m_{\mathrm{c} \imath} / B\right) \cos \left[2 \pi\left(F_{\imath} / B-\gamma_{2}\right)\right]
$$

where $u_{0}=14.694$. The index $i$ refers to $\mathrm{S}_{\imath}$ series; $a_{\imath}$ and $\gamma_{\imath}$ are constants; $m_{\mathrm{c} \imath}$ and $T_{\mathrm{D}}$ are the effective cyclotron mass (in $m_{0}$ units) and the Dingle temperature, respectively. The upper solid line in Figure 2 is the best fit of equation (1) to the data while the lower solid lines are the respective contributions of $S_{1}$ and $S_{2}$ series [17]. Equation (1) is useful, in the present case, mainly in order to determine the value of the phase factor $\gamma_{1}$ for $S_{1}$ series, which otherwise cannot be determined unambiguously (see the middle solid line in Fig. 2). The Landau level index $(n)$ corresponding to each individual oscillation is indicated in Figure 2. However, while the $\gamma$ value related to oscillations belonging to $S_{2}$ series is $\gamma_{2}=0.6 \pm 0.1$ ( .e. definitely in between 0 and 1), $\gamma$ value is close to 0 for $S_{1}\left(\gamma_{1}=0.03\right.$ for the data in Fig. 2). As a consequence, the index of Landau levels for $S_{1}$ series cannot be determined unambiguously. It can nevertheless be noticed that low Landau levels are reached at high magnetic field: down to $n=1$ (or 2 ) for $S_{1}$ series.

It can be noticed in Figure 2 that some discrepancy between equation (1) and the data starts to appear above $\sim 10 \mathrm{~T}$. In the very high field range, the oscillation amplitude of $\mathrm{S}_{2}$ series strongly decreases while an additional series, hereafter referred as $\mathrm{S}_{4}$, appears with 


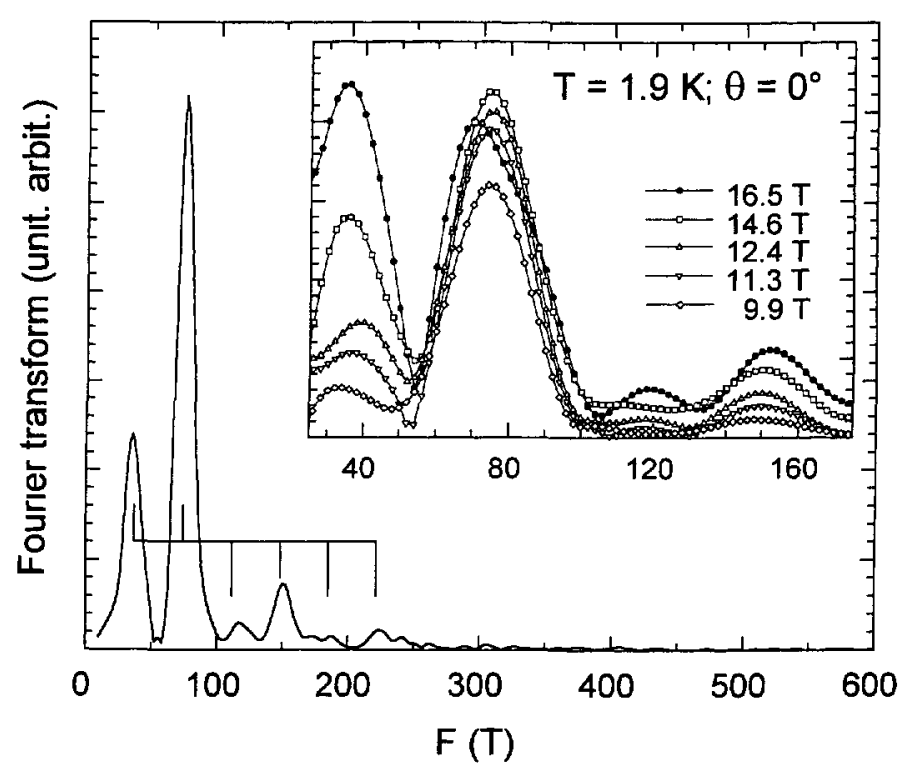

Fig. 3. - Fourier transform of the data in Figure 2. The window field covers the range 5 to $52 \mathrm{~T}$. The inset evidences the magnetic field dependence of the Fourier spectrum. For each curve, the window field width is $\Delta(1 / B)=0.081 \mathrm{~T}^{-1}$ The mean field value is indicated in the figure.

a frequency about twice $F_{2}: F_{4}=(150 \pm 10) \mathrm{T}$. In addition, the $\mathrm{S}_{1}$ oscillation (labelled with $n=1$ in Fig. 2) observed in this high field range is very sharp. This feature might account for the increasing harmonic content in $\mathrm{FT}$ as the mean value of the window field increases (see the inset of Fig. 3).

The angle dependence of the oscillatory part of the magnetoresistance is displayed in Figure 4. First, the above reported change in the oscillation spectrum is still clearly observed as the magnetic field is rotated in the $a^{\prime} b^{*}$ plane up to $\pm 60^{\circ}$ ( $a^{\prime}$ is the direction perpendicular to $c$ in the $a c$-plane). At higher $\theta$ values, the field component normal to the conducting planes is too low in order to evidence the low index Landau levels. The oscillation frequency roughly varies with $\theta$ according to a cosine law (Fig. 5a) in agreement with [10]. However, a thorough examination reveals deviation from the $2 \mathrm{D}$ behaviour since $F_{l} \cos (\theta) / F_{l}(\theta=0)$ (the index $i$ stands for 1, 2 or 4 ) varies with $\theta$ (Fig. 5b). For this reason, data in Figure 4 have been plotted against $F_{2}(\theta) /\left[B F_{2}(\theta=0)\right]$ rather than against $1 /[B \cos (\theta)]$. The observed deviation of the oscillation frequency of all series from cosine law suggests that (BEDO-TTF) ${ }_{2} \mathrm{ReO}_{4} \cdot \mathrm{H}_{2} \mathrm{O}$ is not strongly two-dimensional. It can be noticed that $F_{2}$ remains twice $F_{1}$ in the tilt angle range covered by the experiments, within few percent (Fig. 5c), in agreement with previous data $[10,15]$. On the contrary, the oscillation amplitude of both $\mathrm{S}_{1}$ and $\mathrm{S}_{2}$, as deduced from FT analysis ( $A_{1}$ and $A_{2}$, respectively), varies non-monotonously as $\theta$ varies (Fig. $5 \mathrm{~d}$ ). Furthermore, while $A_{1}$ is very close to $A_{2}$ at high tilt angle, $A_{1}$ is well lower than $A_{2}$ for $\theta=0^{\circ}$.

Effective cyclotron mass have been calculated from the amplitude of FT both for $\theta=0^{\circ}$ and $\theta=50^{\circ}$ (as above mentioned, the latter tilt angle provides larger oscillation amplitude than $\theta=0^{\circ}$ for $S_{1}$ series). In the case of cylindrical FS, the effective cyclotron mass should vary as $m_{c}(\theta)=m_{c} / \cos (\theta)$ [18]. However, as the cosine law is not satisfactorily fulfilled (see Fig. 5b), data for $\theta=50^{\circ}$ have been scaled by the frequency ratio [19] $F_{2}\left(\theta=0^{\circ}\right) / F_{2}\left(\theta=50^{\circ}\right)=0.7$, 


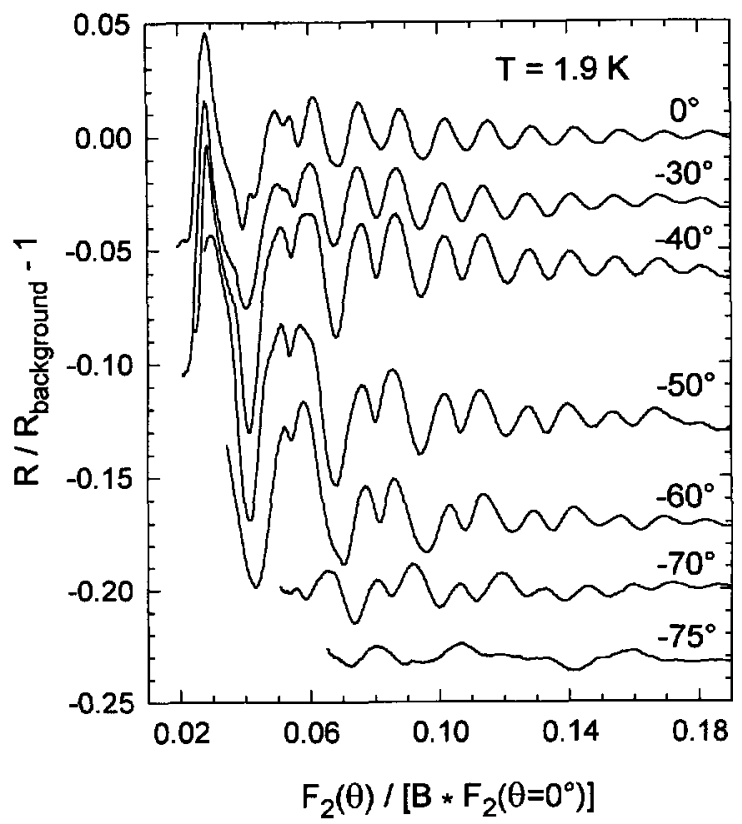

Fig. 4. - Oscillatory part of the magnetoresistance deduced from Figure $1 . R_{\text {background }}$ is the monotonous part of the magnetic field-dependent resistance (see text).

Table I. - Calculated effective cyclotron mass for the different oscillation series. $\theta$ is the $\left(B, b^{*}\right)$ angle.

\begin{tabular}{|l|c|c|c|}
\hline & $\mathrm{S}_{1}$ & $\mathrm{~S}_{2}$ & $\mathrm{~S}_{4}$ \\
\hline$\theta=0^{\circ}[10]$ & $1.15 \pm 0.10$ & $0.9 \pm 0.05$ & \\
\hline$\theta=0^{\circ}$ [this paper] & & $0.76 \pm 0.05$ & $1.3 \pm 0.2$ \\
\hline$\theta=50^{\circ}$ [this paper] & $1.05 \pm 0.15$ & $0.66 \pm 0.07$ & $0.95 \pm 0.10$ \\
\hline
\end{tabular}

according to the data in Figure 5a. The calculated effective cyclotron mass $\left(m_{\mathrm{c}}\right)$ for each oscillation series are given in Table I. No magnetic field-dependence of the effective cyclotron mass is observed in the range covered by the experiments. Effective cyclotron mass for $\mathrm{S}_{1}$ series cannot be calculated for $\theta=0^{\circ}$ due to the low value of the oscillation amplitude for this field direction; for $\theta=50^{\circ}$, a good agreement with [10] is obtained. However, the effective cyclotron mass related to $\mathrm{S}_{2}$ series is found to be slightly lower than in reference [10]. This discrepancy could be due to the different temperature range considered for the calculations: down to $0.5 \mathrm{~K}$ in reference [10] compared to $1.9 \mathrm{~K}$ and $1.7 \mathrm{~K}$ for $\theta=0^{\circ}$ and $50^{\circ}$, respectively in the present case. On the other hand, $m_{\mathrm{c}}$ seems to be slightly angle-dependent for $\mathrm{S}_{2}$ and $\mathrm{S}_{4}$ series. Nevertheless, these findings need to be checked by additional experiments both at lower temperature and other magnetic field orientations, since $m_{\mathfrak{c}}$ is only determined with a quite large uncertainty, in particular in the case of the $\mathrm{S}_{4}$ series. Furthermore, as evidenced in the inset of Figure 3, the harmonic content in the oscillations increases as the magnetic field increases which could hamper the determination of the effective cyclotron mass for this latter series. 


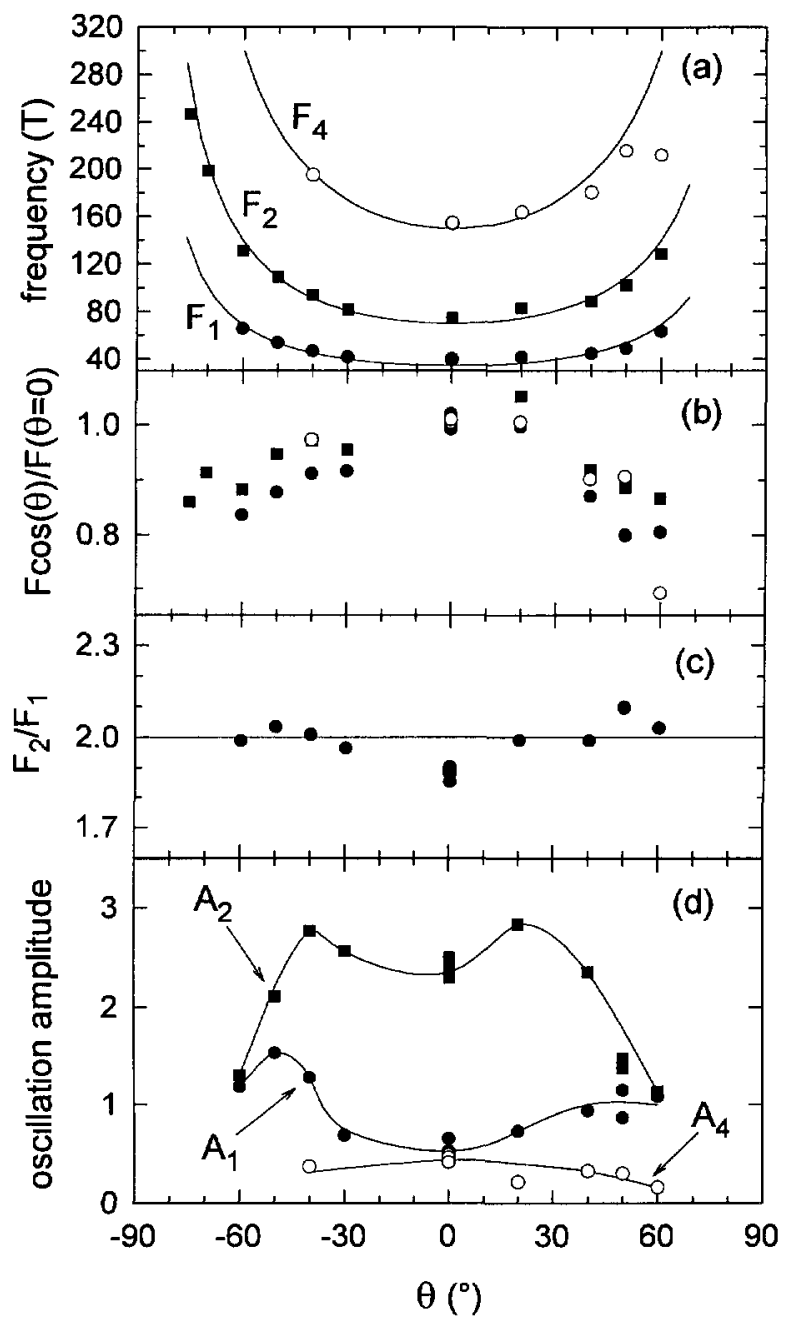

Fig. 5. - Angle $(\theta)$ dependence of parameters relevant to the oscillation series $S_{1}, S_{2}$ and $S_{4}$ (see text). The angle dependence of the oscillation frequency is given in Figure 5a, the deduced scaled values and frequency ratio $F_{2} / F_{1}$ are displayed in Figures $5 \mathrm{~b}$ and $5 \mathrm{c}$, respectively. Oscillation amplitude of the three series, deduced from FT calculations, are collected in Figure 5d. The window field for the calculations of the data for $\mathrm{S}_{4}$ series is within the Landau levels 4 to 12 for all the tilt angle values covered by the experiments. For both $S_{1}$ and $S_{2}$ series, the window field is between Landau levels 3 and 14 of the $\mathrm{S}_{2}$ series (see Fig. 2).

As reported above, the oscillation spectrum changes with magnetic field (see Figs. 2 and 4). This can be further evidenced by a Dingle analysis. Dingle plots are reported both for $\theta=0^{\circ}$ and $\theta=50^{\circ}$ in Figures $6 \mathrm{a}$ and $6 \mathrm{~b}$ respectively, where full lines are the best fits to the data [20]. An anomaly can be observed in the curves of Figure 6 in the field range $(10-14) \mathrm{T}$ whatever the considered oscillation series and field direction. This is in fair agreement with the oscillation spectrum change evidenced in Figures 2 and 4 and is certainly in connection with the negative magnetoresistance observed for $\theta=90^{\circ}$ in Figure 1. These features are likely the signature of a magnetic field-induced phase transition towards a more conducting state. 

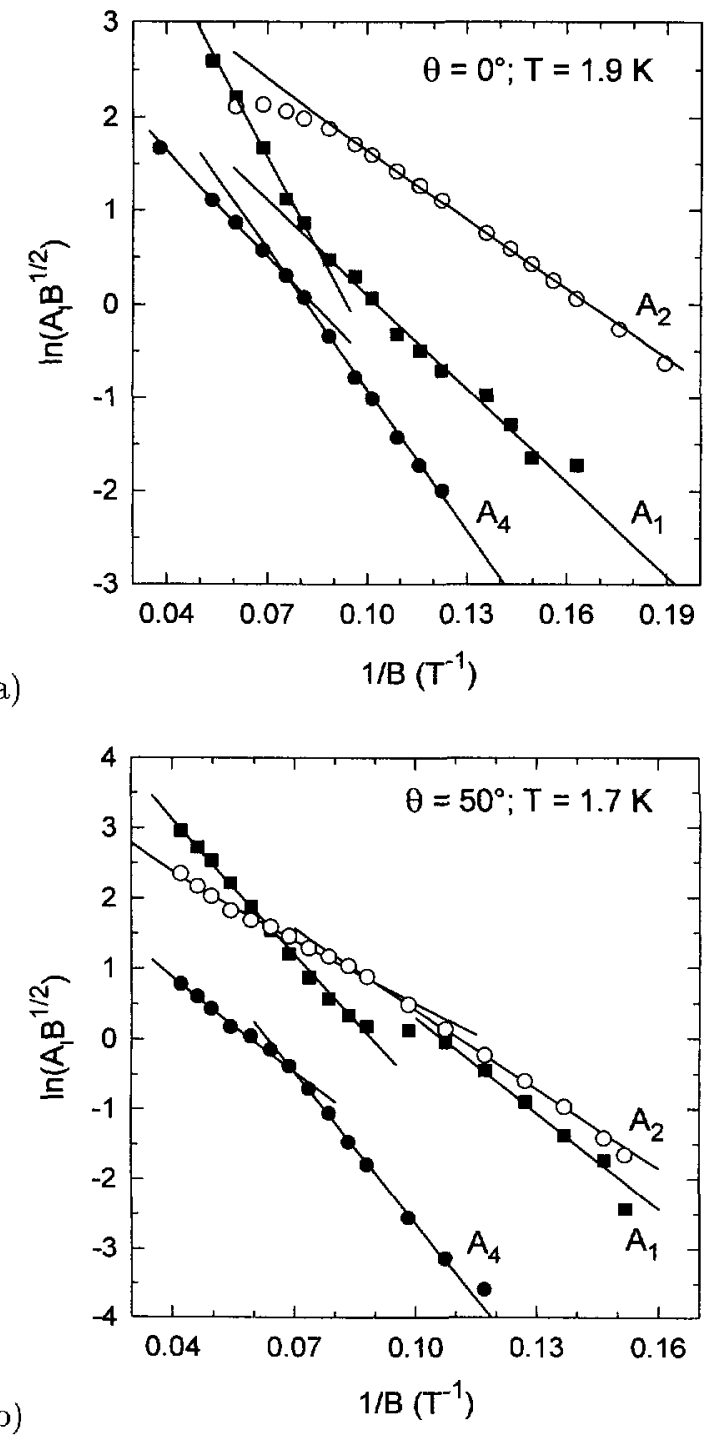

Fig. 6. - Dingle plot for the $S_{1}, S_{2}$ and $S_{4}$ series deduced from data in Figure 4 for $\theta=0^{\circ}$ (Fig. 6a) and $\theta=50^{\circ}$ (Fig. 6b). $A_{2}$ is the oscillation amplitude of the $\mathrm{S}_{2}$ series $(i=1,2,4)$. Each data point has been calculated from Fourier transform amplitude with a window field involving six individual oscillations of the $\mathrm{S}_{2}$ series.

Since the magnetic field range at which the anomaly occurs in Dingle plots does not depend on the tilt angle, it mav be presumed that the magnetic field-induced phase transition, which is the most clearly evidenced in the magnetoresistance data at $\theta=90^{\circ}$, is angle-independent. Assuming that the sample resistance at a given magnetic field can be regarded as due to the additive contributions of the phase transition $\left(\Delta R_{\varphi}(B)\right)$ and orbital effects $\left(\Delta R_{0}(B, \theta)\right)$. the latter being not linked to the phase transition, yields $R(B, \theta)=R(B=0)+\Delta R_{\varphi}(B)+$ $\Delta R_{0}(B, \theta)$. Assuming further the orbital-dependent part of the magnetoresistance depends. 


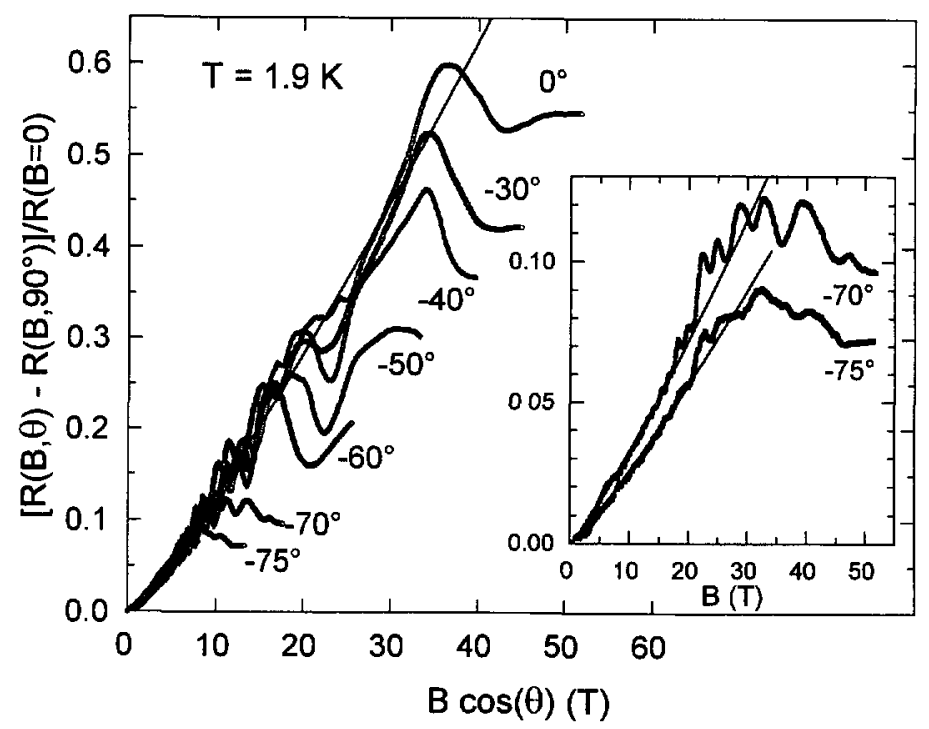

Fig. 7. - Orbital-dependent part of the magnetoresistance versus the component of the magnetic field normal to the conducting planes. The data, deduced from Figure 1, are calculated as $[R(\theta)-R(\theta=$ $\left.\left.90^{\circ}\right)\right] / R(B=0)$. The inset displays the magnetic field-dependence of $\left[R(\theta)-R\left(\theta=90^{\circ}\right)\right] / R(B=0)$ for $\theta=70^{\circ}$ and $75^{\circ}$, respectively. Solid lines are best fits to a power law with exponent $\alpha=1.15$ (see text).

through a power law, on the field component perpendicular to the conducting plane only, yields $\Delta R_{0}(B, \theta) \propto[B \cos (\theta)]^{\alpha}$. It should be noticed that such a power law can only be regarded as a phenomenological approximation of the data. According to the above crude hypotheses, $\Delta R_{\varphi}(B)$ and $\Delta R_{0}(B, \theta)$ can deduced from the magnetoresistance data at $\theta=90^{\circ}$ and $\left[R(B, \theta)-R\left(B, 90^{\circ}\right)\right] / R(B=0)$, respectively. The magnetic field-dependence of the orbital part of the magnetoresistance, deduced from the data in Figure 1, is displayed in Figure 7 where solid lines are the best fits to the data with an exponent $\alpha=1.15$. A good agreement with the data is obtained for magnetic fields up to $\sim 30 \mathrm{~T}$ which confirms that the observed fieldinduced phase transition does not depend on the magnetic field orientation in this field range. However, in magnetic field higher than $\sim 30 \mathrm{~T}$, the power-law cannot account for the data. Such a behaviour could be understood, e.g. if the compound is not perfectly compensated at high magnetic field. In addition, the field component perpendicular to the conducting planes is not the only pertinent parameter to account for the data as a whole in the very high field range. In particular, for high tilt angle values, $\left[R(B, \theta)-R\left(B, 90^{\circ}\right)\right] / R(B=0)$ decreases as the magnetic field increases (see the inset of Fig. 7). This latter feature likely indicates that the magnetic field-induced phase transition depends on the orientation of the magnetic field above $\sim 30 \mathrm{~T}$.

\section{Discussion and Conclusion}

At low magnetic field, two oscillations series, $\mathrm{S}_{1}$ and $\mathrm{S}_{2}$, with frequency $F_{1}$ and $F_{2}$, respectively, are observed, in agreement with previous studies $[10,14,15]$. As it was mentioned in the introduction, $S_{1}$ and $S_{2}$ have been ascribed to electron and hole orbits respectively. Nevertheless, 
both $F_{1}$ and $F_{2}$ frequencies yield cyclotron orbits area lower by a factor of more than two than those predicted for electron and hole orbits by band structure calculations (although the ratio $F_{2} / F_{1}$ amounts to 2 in agreement with calculations). This discrepancy can be understood keeping in mind that band structure calculations rely on crystalline data obtained at room temperature while magnetoresistance data are recorded at low temperature. Indeed, the crystal structure modifications, even if they are very small, linked to the first order metal-metal phase transition at $\sim 210 \mathrm{~K}[11,12]$ might account for a significant change of the FS area. It is also likely that the so called SDW transition leads to a decrease of the orbits area. Moreover, it should be pointed out that, due to the small size of the carriers tubes (few percent of the FBZ) electronic band structure calculations can only yield a rough estimation of the orbits area.

At high magnetic field, the amplitude of $\mathrm{S}_{2}$ oscillations decreases while $\mathrm{S}_{4}$ oscillations rise up with a frequency $F_{4}$ about twice $F_{2}$ which is a signature of a magnetic field-induced phase transition. In the high magnetic field range, where $S_{4}$ series are present, a strong change in the shape of the individual oscillation belonging to $S_{1}$ series (ascribed to Landau level $n=1$ in Fig. 2) can also be noticed. These changes in the oscillations spectrum are likely responsible of the slope change observed in Dingle plots in the range 10-14 $\mathrm{T}$ (see Fig. 6). For magnetic field parallel to the conducting planes, a negative magnetoresistance which saturates at high field is observed above $10 \mathrm{~T}$. This resistance decrease, although it is less clearly observed for other magnetic field directions, is independent of the field direction up to $\sim 30 \mathrm{~T}$.

To account for these phenomena, a magnetic field-induced decondensation of the SDW could be first considered. Indeed, it can be noticed that the oscillation frequency linked to the $\mathrm{S}_{4}$ series corresponds to an orbit area of $3 \%$ of the FBZ which is surprisingly close to the hole FS area predicted by the calculations $(3.4 \%)$. However, it should be noticed that previous magnetoresistance experiments performed under hydrostatic pressure as high as $11 \mathrm{kbar}$ and in magnetic fields applied perpendicularly to the conducting planes have evidenced a transition towards a less conducting state in the temperature range below $\sim 4 \mathrm{~K}$ [15]. This discrepancy, which could be only seeming owing to the different experimental conditions, demonstrates that additional experiments are needed to get a better knowledge of the (magnetic field, temperature, pressure) phase diagram of (BEDO-TTF $)_{2} \mathrm{ReO}_{4} \cdot \mathrm{H}_{2} \mathrm{O}$.

As mentioned in the introduction, a field-induced decondensation of the carriers involved in a $\mathrm{SDW}$ has also been reported in the $\alpha-(\mathrm{BEDT}-\mathrm{TTF})_{2} \mathrm{KHg}(\mathrm{SCN})_{4}$ compound [8]. However, the FS topology is very different in the latter compound when compared to (BEDO-TTF) ${ }_{2} \mathrm{ReO}_{4}$. $\mathrm{H}_{2} \mathrm{O}$. Indeed, while the SDW transition only involves the $1 \mathrm{D}$ sheets in the $\alpha$-phase compound, only small $2 \mathrm{D}$ closed orbits are present in (BEDO-TTF $)_{2} \mathrm{ReO}_{4} \cdot \mathrm{H}_{2} \mathrm{O}$ according to calculations [10]. On the other hand, the oscillatory behaviour both of (BEDO-TTF) ${ }_{2} \mathrm{ReO}_{4} \cdot \mathrm{H}_{2} \mathrm{O}$ and the Bechgaard salt (TMTSF) ${ }_{2} \mathrm{NO}_{3}$ bear striking similarities. Indeed, a low frequency oscillations series $\left(F_{1}=63 \mathrm{~T}\right)$ is observed at low field in the latter case, which is replaced by a high frequency series $\left(F_{\mathrm{h}}=247 \mathrm{~T}\right)$ above $\sim 16 \mathrm{~T}$ [21]. This latter series is known as the "fast oscillations" series which occurs in Bechgaard salts even in the case where the ground state is insulating. It should be noticed that, due to the anion ordering, the wave vector of which is parallel to the high conductivity axis, the FS of (TMTSF) ${ }_{2} \mathrm{NO}_{3}$ is composed of one electron and one hole $2 \mathrm{D}$ orbits so that it can be regarded, as in the present case, as a compensated $2 \mathrm{D}$ semimetal. However, (i) contrariwise to the case of (BEDO-TTF) ${ }_{2} \mathrm{ReO}_{4} \cdot \mathrm{H}_{2} \mathrm{O}$ (in the magnetic field range lower than $\sim 30 \mathrm{~T}$ ), only the component of a magnetic field, parallel to the lowest conductivity axis, is effective on the SDW state and (ii) it stabilises the SDW state of (TMTSF) ${ }_{2} \mathrm{NO}_{3}$ [4].

According to the presently available FS calculations, the gap between the hole and the two electron orbits is rather small. It can then be also suggested that $\mathrm{S}_{4}$ series arises from 
magnetic breakdown, provided the gap between the orbits remains small in the SDW state. Such a picture would reconcile the present data with the results of reference [15]: at low temperature a magnetic field lower than $12 \mathrm{~T}$ stabilizes the SDW state while a magnetic breakdown phenomenon starts to appear at high magnetic field. Nevertheless, this hypothesis strongly need to be checked. In that respect, reliable band structure calculations based on crystallographic data recorded at low temperature would be very useful.

\section{Acknowledgments}

We would like to thank E. Canadell for very fruitful discussions, S. Askenazy and E.B. Yagubskii for their interest and encouragements. We also acknowledge INTAS for financial support within the contract No 93-2400. V.N.L. is grateful to Spanish Ministerio de Education y Ciencia for Sabbatical at ICMAB-CSIC, Barcelona. The work was supported in part in the framework of the project $\sharp 93030$ within the Russian Program "Topical Aspects of the Condensed Matter Physics" and supported by Scientific Council "Superconductivity".

\section{References}

[1] For a review, see e.g. Proceedings of the International Conference on Science and Technology of Synthetic Metals, Seoul, Korea. 1994 (Synth. Met. 69-71 (1995)) and references therein.

[2] Biskup N., Tomic S. and Jérome D., Phys. Rev. B 51 (1995) 17972; Danner G.M., Chaikin P.M. and Hannahs S.T., Phys. Rev. B 53 (1996) 2727.

[3] For a review, see e.g. Low Dimensional Conductors and Superconductors, D. Jérome and L. Caron, Eds. (NATO Advanced Study Institute, Ser. B) Vol. 55 (Plenum New York, 1987).

[4] Audouard A. and Askenazy S., Phys. Rev. B 52 (1995) R700.

[5] Montambaux G., Phys. Rev. B. 38 (1988) 4788.

[6] Bjelis A. and Maki K., Phys. Rev. B 45 (1992) 12887.

[7] Klepper J., Brooks J.S., Chen X., Bradaric I., Tokumoto M., Kinoshita N., Tanaka Y. and Agosta C.C., Phys. Rev. B 48 (1993) 9913.

[8] Caulfield J., Blundell S.J., du Croo de Jongh M.S.L., Hendricks P.T.J., Singleton J., Doporto M., Pratt F.L., House A., Perenboom J.A.A.J. and Hayes W.. Phys. Rev. B 51 (1995) 8325.

[9] House A., Harrison N., Blundell S.J., Deckers I., Singleton J., Herlach F., Hayes W., Perenboom J.A.A.J., Kurmoo M. and Day P., Phys. Rev. B 53 (1996) 9127.

[10] Kahlich S., Schweitzer D., Rovira C., Paradis J.A., Whangbo M.H., Heinen I., Keller H.J., Nuber B., Bele P., Brunner H. and Shibaeva R.P., Z. Phys. B 94 (1994) 39.

[11] Kahlich S., Schweitzer D., Heinen I., Lan S.E., Nuber B., Keller H.J., Winzer K. and Helberg H.W., Solid State Commun. 80 (1991) 191.

[12] Buravov L.I., Khomenko A.G., Kushch N.D., Laukhin V.N., Schegolev A.I., Yagubskii E.B., Rozenberg L.P. and Shibaeva R.P., J. Phys I France 2 (1992) 529.

[13] Kahlich S., Schweitzer D., Auban-Senzier P., Jérome D. and Keller H.J.. Solud State Commun. 83(1992) 77.

[14] Kovalev A.E., Pesotskii S.I., Gilevski A. and Kushch N.D., JETP Lett. 59 (1994) 560 [Pis'ma v Zh. Eksp. Teor. Fiz. 59 (1994) 530]. 
[15] Audouard A., Auban-Senzier P., Laukhin V.N., Brossard L., Jérome D. and Kushch N.D., Europhys. Lett. 34 (1996) 599.

[16] Shibaeva R.P. and Zavodnik V.E., Kristallografiya 38(1993) 114 (in Russian).

[17] The fit of the data in Figure 2 is obtained assuming $u_{0} T m_{\mathrm{c} 2} / B$ is large when compared to unity. The values of $F_{\imath}$ and $\left(T+T_{\mathrm{D}}\right) m_{\mathrm{c} \imath} / B$ are deduced for both series from FT (see Fig. 3) and Dingle plots in the low field range (see below, Fig. 6a) respectively. The remaining free parameters are $a_{2}$ and $\gamma_{2}$.

[18] Wosnitza J., Crabtree G.W., Wang H.H., Vashon M.D. and Williams J.M., Phys. Rev. Lett. 67 (1991) 263.

[19] Doporto M., Pratt F.L., Singleton J., Kurmoo M. and Hayes W., Phys. Rev. Lett. 69 (1992) 991.

[20] Dingle temperature values can only been determined with a large uncertainty, mainly due to the rather large uncertainty on the calculated value of $m_{\mathrm{c}}$. Moreover, such parameter is not actually relevant for the discussion of the results.

[21] Audouard A., Goze F., Ulmet J.P., Brossard L., Askenazy S. and Fabre J.M., Phys. Rev. $B 50$ (1994) 12726. 